\title{
DIAGNÓSTICO GENÉTICO E CLÍNICO DO AUTISMO INFANTIL
}

\author{
MARIA IONE FERREIRA DA COSTA*, HENRIQUE GIL DA SILVA NUNESMAIA**
}

\begin{abstract}
RESUMO - Os principais objetivos deste estudo foram caracterizar variáveis selecionadas para um melhor entendimento e diagnóstico do autismo infantil, tais como: achados clínicos e de imagem, critérios diagnósticos, frequência de distúrbios neuropsiquiátricos nos familiares dos propósitos, recorrência familiar e a ocorrência de consanguinidade entre os pais dos propósitos e entre outros casais da família. A amostra foi constituída de 36 propósitos, de ambos os sexos e na faixa etária de 1 a 20 anos, pertencentes a 35 famílias distintas, todos com diagnóstico clínico de autismo infantil. Os resultados foram os seguintes: a deficiência mental foi observada clinicamente em todos os pacientes da amostra e convulsão em 27,8\%; distúrbios neuropsiquiátricos foram referidos em pelo menos um familiar dos propósitos (97,14 \% das famílias), autismo recorrente em 11,42 \% e consanguinidade nos pais (11,42\%), avós e bisavós (2,86 \%); achados anormais de tomografia computadorizada de crânio foram verificados em três propósitos. $\mathrm{O}$ conjunto destes resultados reforça a sugestão do modelo de herança multifatorial com limiar diferencial para sexo no autismo infantil. A avaliação clínica de todo caso de autismo infantil deverá contemplar sempre os aspectos neurológicos, psiquiátricos e genéticos.
\end{abstract}

PALAVRAS-CHAVE: autismo infantil, deficiência mental, distúrbios neuropsiquiátricos.

\section{Genetic and clinical diagnosis of infantile autism}

ABSTRACT - The main objectives of this study were to characterize the selected variables for a better understanding and diagnosis of infantile autism such as clinical and image findings, diagnostic criteria, frequency of neuropsychiatric disorders in the subjects' families, familial recurrence and occurrence of consanguinity between the subjetcs' parents and between other couples in the family. The sample was composed of 36 subjects of both sexes, in the age group from 1 through 20 years old, members of 35 distinctive families, all of which presenting clinical diagnosis for infantile autism. Mental retardation was clinically observed in all subjects of the sample and convulsion in $27.8 \%$; neuropsychiatric disorders were referred in at least one family member of the subjects ( $97.14 \%$ of the families); recurrent autism in $11.42 \%$ grandparents and great-grand-parents $(2.86 \%)$; abnormal findings in computed tomography scan were observed in three subjects. These results support the suggestion of the multifactorial heredity model with differential threshold fold sex in infantile autism. The clinical evaluation of all infantile autism cases should always appraise the neurological, psychiatric and genetic features.

KEY WORDS: infantile austim, mental retardation, neuropsychiatric disorders.

O autismo infantil é uma severa desordem da personalidade, que se manifesta na infância precoce por um anormal desenvolvimento de linguagem e relações com os outros ${ }^{15}$. No Manual Diagnóstico e Estatístico de Transtornos Mentais (DSM IV ${ }^{\mathrm{TM}}$ ) da Associação Americana de Psiquiatria $(\mathrm{AAP})^{2}$, está classificado no subgrupo denominado "Transtornos Invasivos do Desenvolvimento". Este subgrupo é caracterizado por severas deficiências e prejuízo invasivo em múltiplas áreas do desenvolvimento, incluindo perdas na interação social recíproca e na comunicação, apresentando

Centro de Estudos e Projetos em Saúde Materno-Infantil - Departamento Materno-Infantil-CCS Universidade Federal da Paraíba: *Médica do Centro de Reabilitação Infantil - Secretaria de Saúde do Estado do Rio Grande do Norte - Natal-RN; **Professor do Departamento Materno-Infantil - CCS - Universidade Federal da Paraíba - João Pessoa-PB. Aceite: 1-outubro-1997.

Dra Maria Ione Ferreira da Costa - Rua Ponta de Serrambi 2249, Conjunto Ponta Negra - 59092-170 Natal RN Brasil. FAX 0842116951. 
comportamentos, interesses e atividades estereotipadas. Ornitz ${ }^{17}$ descreve uma evolução para essa doença (Quadro 1), situando no tempo os sintomas dessas crianças. Segundo Leboyer ${ }^{13}$, é fundamental ter uma visão dinâmica da evolução da sintomatologia autística, principalmente em função da idade do paciente.

O diagnóstico do autismo infantil é baseado principalmente no quadro clínico do paciente, não havendo ainda um marcador biológico que o caracterize. No entanto, Herault et al. ${ }^{11}$ com o objetivo de confirmar uma associação entre autismo e marcadores do oncogene C, Harvey-ras (HRAS), estudaram o genótipo de um grupo de crianças autistas, bem caracterizadas clínica e geneticamente $(n=55)$, em comparação com um grupo controle de crianças normais $(n=55)$. Encontraram diferenças significantes nas frequências alélicas entre as duas populações, de dois marcadores do gene HRAS localizadas no braço curto do cromossomo 11, sugerindo que esta região do DNA no cromossomo 11 confere susceptibilidade para o autismo infantil. $\mathrm{O}$ achado faz antever, para um futuro próximo, a utilização de marcadores genéticos na avaliação clínica da doença.

Na década de 80, escalas, questionários e critérios foram introduzidos, com o objetivo tentar uniformizar esse diagnóstico ${ }^{1,9,12,19}$. Os critérios diagnósticos do Manual de Diagnóstico e Estatística de Distúrbios Mentais, da AAP (DSM III-R, versão 1987) ${ }^{1}$, foram os mais utilizados na literatura internacional até $1993^{20}$. Recentemente, foi editada a quarta versão do DSM apresentando critérios diagnósticos mais detalhados do autismo infantil ${ }^{2}$.

Quadro 1. Características clínicas evolutivas detectadas por período do desenvolvimento da criança com austimo infantil (*).

\begin{tabular}{|c|c|}
\hline Período do desenvolvimento & Características clínicas \\
\hline Recém-nascido & $\begin{array}{l}\text { - parece diferente dos outros bebês } \\
\text { - parece não precisar de sua mãe } \\
\text { - raramente chora ("um bebê muito comportado") } \\
\text { - torna-se rígido quando é pego no colo } \\
\text { - às vezes muito reativo aos elementos e irritável }\end{array}$ \\
\hline Primeiro Ano & $\begin{array}{l}\text { - não pede nada, não nota sua mãe } \\
\text { - } \text { sorrisos, resmungos, respostas antecipadas são ausentes ou retardados } \\
\text { - falta de interesse por jogos, muito reativo aos sons } \\
\text { - não afetuoso } \\
\text { - não interessado por jogos sociais } \\
\text { - quando é pego no colo, é indiferente ou rígido } \\
\text { - ausência de comunicação verbal ou não verbal } \\
\text { - hipo ou hiper-reativo aos estímulos } \\
\text { - aversão pela alimentação sólida } \\
\text { - etapas do desenvolvimento motor irregulares ou retardadas }\end{array}$ \\
\hline Segundo e o Terceiro Anos & $\begin{array}{l}\text { - indiferente aos contatos sociais } \\
\text { - comunica-se mexendo a mão do adulto } \\
\text { - o único interesse pelos brinquedos, consiste em alinhá-los } \\
\text { - intolerância à novidade nos jogos } \\
\text { - procura estimulações sensoriais como ranger os dentes, esfregar e } \\
\text { arranhar superfícies, fitar fixamente detalhes visuais, olhar mãos em } \\
\text { movimentos ou objetos com movimentos circulares. } \\
\text { - particularidade motora: bater palmas, andar na ponta dos pés, balançar a } \\
\text { cabeça, girar em torno de si mesmo }\end{array}$ \\
\hline Quarto e o Quinto Anos & $\begin{array}{l}\text { - ausência do contato visual } \\
\text { - jogos: ausência de fantasias, de imaginação, de jogos de representação } \\
\text { - linguagem limitada ou ausente - ecolalia - inversão pronominal } \\
\text { - anomalias do ritmo do discurso, do tom e das inflexões } \\
\text { - resistência às mudanças no ambiente e nas rotinas }\end{array}$ \\
\hline
\end{tabular}

(*) Adaptado de Ornitz ${ }^{17}$ e Leboyer ${ }^{13}$. 
Os principais objetivos deste estudo foram caracterizar variáveis selecionadas para um melhor entendimento e diagnóstico da doença, tais como: achados clínicos e de imagem, critérios diagnósticos, frequência de distúrbios neuropsiquiátricos nos familiares dos propósitos, recorrência familiar e ocorrência de consanguinidade entre os pais dos propósitos e entre outros casais da família.

\section{CASUÍSTICA E MÉTODOS}

A amostra foi constituída de 36 propósitos, de ambos os sexos e na faixa etária de 1 a 20 anos, pertencentes a 35 famílias distintas, todos com diagnóstico clínico de autismo infantil. Os propósitos foram averiguados no período de maio-1993 a julho-1994 em instituições especializadas de três estados da região Nordeste do Brasil, a saber: Fundação Centro Integrado de Apoio ao Portador de Deficiência (FUNAD) e Centro de Educação Especial da Paraíba, na cidade de João Pessoa-PB; Associação de Pais e Amigos dos Excepcionais (APAE) e Serviço de Psicologia Aplicada da Universidade Federal do Rio Grande do Norte (SEPA), na cidade de NatalRN; e Grupo de Atendimento Psico-pedagógico (GAPP), na cidade do Recife-PE.

Foram realizadas entrevistas para preenchimento de: a) formulário de identificação dos propósitos; b) formulário de confirmação diagnóstica, constando de ficha guia com os critérios para Distúrbios Autista do DSM III-R ${ }^{1}$. Os propósitos confirmados foram submetidos a avaliação clínica e genética, constando de história clínica, exame físico e construção do heredograma, sendo as informações anotadas no formulário de avaliação clínica. Os dados foram analisados por estatística descritiva ou comparados pelo teste $Z$.

Foram considerados "Distúrbios Neuropsiquiátricos" o conjunto de patologias referidas, de natureza neurológica e psicopatológica, registradas no Código Internacional de Doenças - $10^{\mathrm{a}}$ edição ${ }^{16}$ tais como: Alcoolismo F10.9, Retardo Mental F79, Doença Convulsiva G40.9. Além disso, foi incluído neste grupo, um subgrupo denominado Psicopatologia Grave F99, para todo transtorno mental não especificado, acompanhado de internamento hospitalar.

\section{RESULTADOS}

A distribuição dos propósitos quanto ao sexo, mostrou uma frequência do sexo masculino $(77,8 \%)$ três vezes maior do que a do sexo feminino (22,2 \%), sendo a razão de sexo de 3,5.

A deficiência mental foi observada clinicamente em todos os pacientes da amostra, em graus variados, de leve, moderado e grave (Tabela 1). A regressão da fala foi o segundo achado clínico mais frequente $(77,8 \%)$ seguido das manchas "café com leite" (36,1\%). Foi relatada a ocorrência de convulsão em $27,8 \%$ dos propósitos, tendo sido episódios ou crises atualmente controladas com o tratamento anticonvulsivante.

O antecedente médico encontrado nas famílias dos pacientes com autismo infantil, com maior frequência (97,14\%), foi o de "Distúrbios Neuropsiquiátricos", referidos em pelo menos um familiar de cada propósito (Tabela 2). O grupo definido como "Psicopatologia grave" foi o mais frequente (62,80\%), seguido de Alcoolismo e Retardo Mental, referidos em 54,3\% e 37,10\% das famílias, respectivamente (Tabela 3). O autismo infantil foi recorrente em quatro famílias (Tabela 2).

A consanguinidade foi verificada em $11,42 \%$ dos pais dos propósitos e em 2,86\% dos avós e bisavós destes.

A tomografia computadorizada de crânio (TCC) foi possível de ser realizada em três propósitos. No propósito da família 25 mostrou cisto porencefálico (Fig 1), referido como cisto benigno, congênito e com frequência associado a hidrocefalia. No propósito da família 28 o achado tomográfico foi o de uma lesão hipodensa na região parietal direita (Fig 2), sugestiva também de cisto porencefálico. No propósito da família 33 a tomografia revelou calcificação do globo pálido, bilateral (Fig 3).

Os propósitos estão distribuídos por número de critérios diagnósticos, em que todos apresentaram pontuação maior do que o número mínimo exigido pelo DSM III-R, que é 8, verificandose que a maior parte dos pacientes, ou seja, 22 propósitos $(61,1 \%)$ apresentaram acima de 14 critérios e 6 destes apresentaram o número máximo de 16. 
Tabela 1. Distribuição dos pacientes com autismo infantil $(n=36)$, por principais achados clínicos.

\begin{tabular}{llll}
\hline Achados & & $\mathrm{n}$ & $\%$ \\
\hline \multirow{2}{*}{ Neurológicos: } & Atraso motor & 10 & 27,8 \\
& Doença convulsiva & 10 & 27,8 \\
& Regressão na fala & 28 & 77,8 \\
& Linguagem ecolálica & 8 & 22,2 \\
& Deficiência mental & 36 & 100 \\
Dermatológicos: & Manchas hipocrômicas & 3 & 8,3 \\
& Manchas "café-com-leite" & 13 & 36,1 \\
Esqueléticos: & Anomalias esqueléticas & 2 & 5,5 \\
\hline
\end{tabular}

Tabela 2. Distribuição de famílias dos pacientes com autismo infantil $(n=35)$ por antecedentes patológicos referidos em pelo menos um familiar.

\begin{tabular}{llcc}
\hline Antecedente Patológico & CID-10* & \multicolumn{2}{c}{ Famílias } \\
\cline { 3 - 4 } & & $\mathrm{n}$ & $\%$ \\
\hline Distúrbios neuropsiquiátricos & & 34 & 97,14 \\
Doença cardíaca & 121.9 & 18 & 62,80 \\
Acidente vascular cerebral & 167.9 & 3 & 81,40 \\
Síndrome de Down & Q90.9 & 3 & 8,50 \\
Malformação congênita & Q07.9 & 2 & 5,70 \\
Doença de Parkinson & G20 & 1 & 2,90 \\
Doença de Alzheimer & G30.9 & 4 & 11,42 \\
Autismo infantil recorrente & F84.0 & & \\
\hline
\end{tabular}

*OMS ${ }^{16}$

Tabela 3. Distribuição de famílias dos pacientes com autismo infantil $(n=35)$ por tipo de distúrbio neuropsiquiátrico referido em pelo menos um familiar.

\begin{tabular}{lccc}
\hline Distúrbio & CID-10 & \multicolumn{2}{c}{ Famílias } \\
\cline { 3 - 4 } & & $\mathrm{n}$ & $\%$ \\
\hline Psicopatologia grave & F 99 & 22 & 62,8 \\
Alcoolismo & F 10,9 & 19 & 54,3 \\
Retardo mental & F 79 & 13 & 37,1 \\
Doença convulsiva & G40,9 & 8 & 22,8 \\
Suicídio & F32,2 & 6 & 17,1 \\
Demência senil & F03 & 3 & 8,5 \\
Outros distúrbios do comportamento & F69 & 5 & 14,3 \\
\hline
\end{tabular}

No estudo dos familiares destacamos três famílias (Fig 4), pela riqueza de informações; a família 7 ilustra a ocorrência de gêmeos concordantes para o autismo infantil, a família 29 a recorrência familiar de autismo infantil e a família 35 a ocorrência de consanguinidade dupla entre os pais do propósito. Além disso, essas três famílias, ilustram a concentração familiar de Distúrbios Neuropsiquiátricos. 


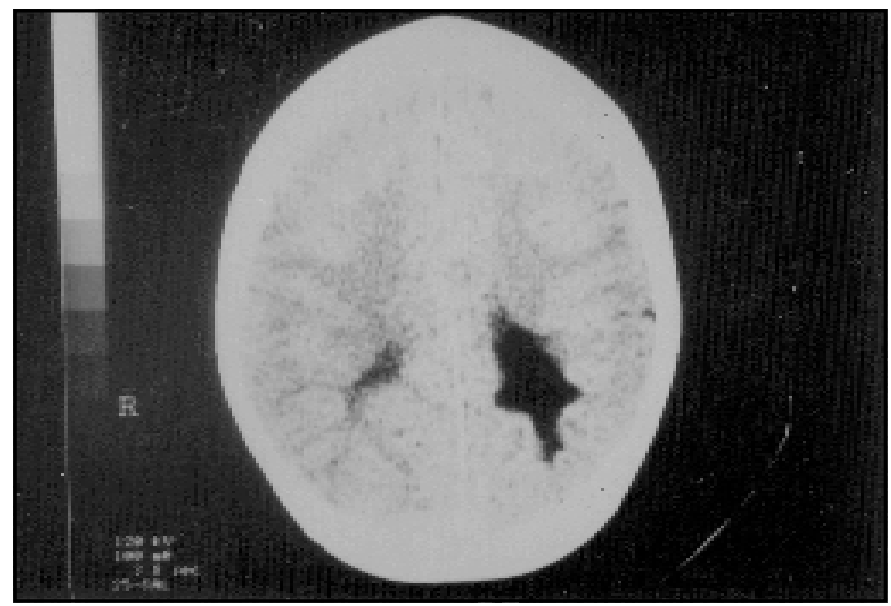

Fig 1. Tomografia computadorizada do crânio do propósito da família 25, apresentando cisto porencefálico localizado no hemisfério cerebral esquerdo.

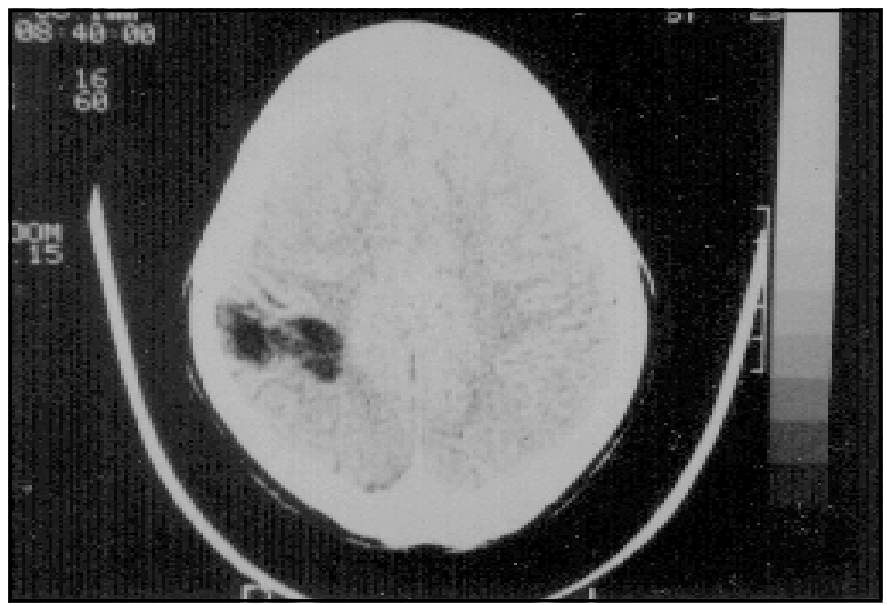

Fig 2. Tomografia computadorizada do crânio do propósito da família 28, sugerindo cisto porencefálico na região parietal direita.

\section{DISCUSSÃO}

De acordo com Clark ${ }^{6}$ aproximadamente $25 \%$ dos afetados com autismo infantil, eventualmente apresentam doença convulsiva. No presente estudo, $27,8 \%$ da amostra foram referidos com episódios de convulsão.

Apesar da frequência elevada de manchas "café com leite" (36,1\%), não foram detectadas, no presente estudo, displasias neurocutâneas associadas. O significado genético dessas manchas é sugestivo de algo acontecendo precocemente no desenvolvimento embrionário a nível do ectoderma, já que genodermatoses como a esclerose tuberosa e a neurofibromatose são condições clínicas que têm sido associadas ao autismo infantil ${ }^{20}$.

Quando comparamos a prevalência de distúrbios neuropsiquiátricos específicos entre os familiares dos propósitos da amostra, de três gerações em média, com as prevalências estimadas na população geral para o alcoolismo ${ }^{18}$, para o retardo mental ${ }^{5}$ e para doença convulsiva ${ }^{14}$, as diferenças encontradas 


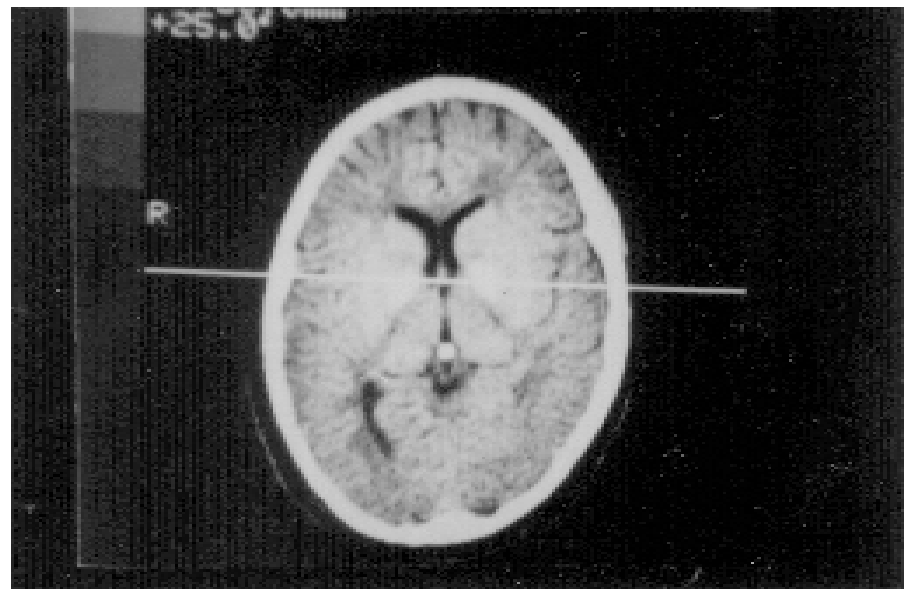

Fig 3. Tomografia computadorizada do crânio do propósito da família 33, apresentando calcificação de globo pálido bilateral.

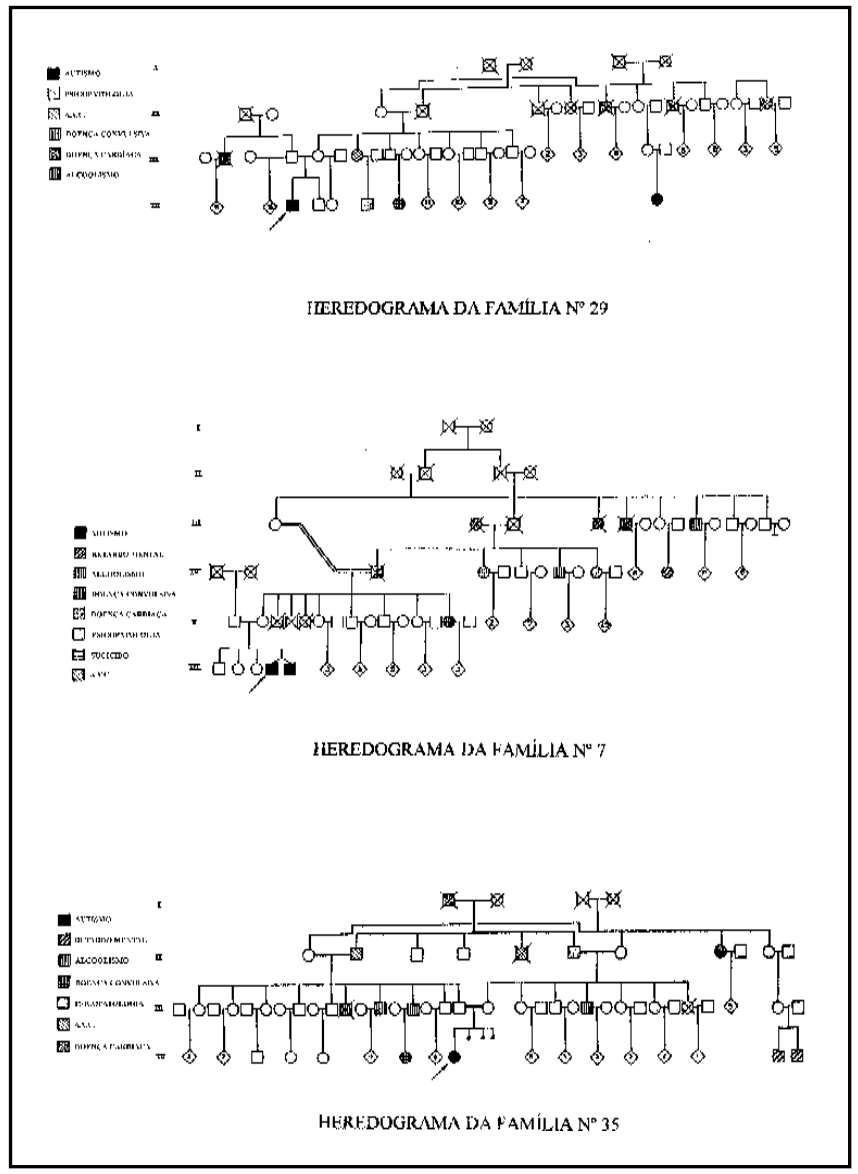

Fig 4. Heredogramas das famílias 7, 29 e 35. 
Tabela 4. Estimativa da prevalência de distúrbios neuropsiquiátricos específicos em familiares de pacientes com autismo infantil e na população geral.

\begin{tabular}{lccc}
\hline \multirow{2}{*}{ Distúrbios } & \multicolumn{2}{c}{ Prevalência por 1000} & \\
\cline { 2 - 2 } & Familiares & População Geral & Teste Z \\
\hline Alcoolismo & $0,320^{1}$ & $0,350^{2}$ & $0,75 \mathrm{NS}$ \\
Retardo mental & $0,152^{1}$ & $0,250^{3}$ & $2,08 \mathrm{SI}$ \\
Doença convulsiva & $0,130^{1}$ & $0,050^{4}$ & $3,15 \mathrm{SI}$ \\
\hline
\end{tabular}

(1) Presente Estudo; (2) Santana ${ }^{18}$; (3) Camp e Kozieski ${ }^{5}$; (4) Mazet e Lebovici ${ }^{14}$

NS, não significante; SI, significante.

(Tabela 4) foram estatisticamente significantes no retardo mental $(Z=2,08)$ e na doença convulsiva ( $Z$ $=3,15)$, não havendo diferença significante para o alcoolismo $(Z=0,75)$. Estes resultados reafirmam a hipótese de maior concentração dos distúrbios neuropsiquiátricos específicos entre os familiares dos pacientes com autismo infantil. Este fato é de particular interesse se levarmos em consideração que a frequência de retardo mental é de $75 \%$ e de doença convulsiva é de $25 \%$ nos pacientes de autismo infantil ${ }^{6}$.

Em resumo, os resultados do presente estudo sugerem que as famílias dos propósitos possuem um perfil psicopatológico característico, já que mostram maior concentração de portadores de distúrbios neuropsiquiátricos do que a população geral. Estudos dirigidos devem ser realizados para explorar este contexto genético-familiar.

No presente estudo, a recorrência familiar foi de $11,42 \%$, confirmando o achado de Freeman et al. ${ }^{8}$, que referem a ocorrência de familiares com múltiplas incidências de autismo infantil, sendo $11,29 \%$ a proporção de famílias com autismo recorrente.

A proporção de casamentos consanguíneos, entre os pais dos propósitos do presente estudo, foi superior $(11,42 \%)$ ao da população geral do Nordeste $(2,00 \%)$, o que não ocorreu com a proporção encontrada entre os avós e bisavós dos pacientes $(2,86 \%)$. A recorrência familiar e o percentual de consanguinidade entre os pais dos propósitos, acima do esperado, sugerem a hipótese de herança multifatorial, já que o endocruzamento aumenta também a probabilidade de ocorrência das doenças multifatoriais.

Bauman e Kemper ${ }^{3}$ mostraram alterações morfológicas na fossa craniana posterior, com alongamento do $4^{\circ}$ ventrículo, a partir de autópsias de pacientes com autismo infantil. Estes achados anatomopatológicos foram confirmados por exames de TCC, por Bauman et al. ${ }^{4}$; e por estudos de imagens de ressonância magnética (IRM), por Gaffney et al. ${ }^{10}$. O estudo de imagem é importante não só na fase de diagnóstico inicial como também na de acompanhamento, para melhor caracterização do achado neurológico.

As proporções dos sinais clínicos, avaliados pelo DSM III-R, apresentaram-se uniformemente distribuídas, com elevado número de critérios, confirmando a eficiência do DSM III-R e o diagnóstico dos pacientes da amostra. É recomendável, nos casos suspeitos de autismo infantil, a utilização dos critérios diagnósticos da $\mathrm{AAP}^{2}$.

Os achados clínicos e genéticos-familiais da amostra confirmam os achados da literatura. Além disso, o conjunto destes resultados reforça a sugestão do modelo de herança multifatorial com limiar diferencial para sexo no autismo infantil.

A principal recomendação de ordem prática, derivada dos resultados do presente estudo, é a de que a avaliação clínica de todo caso de autismo infantil deverá contemplar sempre os aspectos neurológicos, psiquiátricos e genéticos. 
Agradecimentos - Agradecemos aos colegas Roberto Ney Santos Batista, Ozías Arruda de Assis Neto e Maricélia Rodrigues, responsáveis pela realização das tomografias computadorizadas de crânio, apresentadas neste estudo.

\section{REFERÊNCIAS}

1. Associação Americana de Psiquiatria. Manual de estatística e diagnóstico dos distúrbios mentais (DSM III-R). 3. Ed. São Paulo: Manole, 1989.

2. Associação Americana de Psiquiatria. Manual de estatística e diagnóstico de transtornos mentais (DSM IV ${ }^{\mathrm{TM}}$ ). 4. Ed. Porto Alegre: Artes Médicas, 1995.

3. Bauman M, Kemper TL. Histoanatomic observation of the brain in early infantile autism. Neurology 1985;35:866-874.

4. Bauman ML, Lemay ML, Bauman RA, Rosenberger PB. Computerized tomographic observations of the posterior fossa in early infantile autism. Neurology 1985;35:247.

5. Camp BW, Kozieski EB. Developmental disorders. In Hay WW, Groothuis JR, Hayward AR, Levin MJ (eds). Pediatric diagnosis and treatment. 12 Ed. London: Prentice Hall, 1995.

6. Clark BR. Psychosocial aspects of pediatrics and psychiatric disorders. In: Hay WW, Groothuis, JR Hayward AR, Levin MJ (eds.). Pediatric diagnosis and treatment. 12 Ed., London: Prentice Hall, 1995.

7. Daniel WW. Biostatistics: a foundation for analysis in the health sciences. New York: John Wiley, 1974.

8. Freeman BJ, Ritvo ER, Mason-Brothers A, et al. Psychometric assessment of first-degree relatives of 62 autistic probands in Utah. Am J Psychiatry 1989;146:361-364

9. Freeman BJ, Schroth PC, Ritvo E, Guthrie D, Wake L. The behavior observation scale for autism (BOS): initial results of factor analyses. J Autism Dev Disord 1980;10:343-347.

10. Gaffney GR, Kuperman S, Tsai LY, Minchin S, Hassanein KM. Mid sagittal magnetic resonance imaging of autism. Br J Psychiatry 1987;151:831-833.

11. Hérault J, Petit E, Martineau J, et al. Autism and genetics: clinical approach and association study with two markers of HRAS gene. Am J Med Genet 1995;60:276-281.

12. Krug DA, Arick J, Almond P, Behavior Checklist for identifying severely handicapped individuals with high levels of autistic behavior. J Child Pychol Psychiatry 1980;21:221-229.

13. Leboyer M. Autismo infantil. Campinas: Papirus, 1987.

14. Mazet P, Lebovici S. Autismo e psicoses da criança. Porto Alegre: Artes Médicas, 1991.

15. McGillivray BC, Herbst DS, Dill FJ, Sandercock HJ, Tischler B. Infatile autism: an occasional manifestation of fragile $\mathrm{X}$ mental retardation. Am J Med Genet 1986;23:325-358.

16. Organização Mundial de Saúde. Classificação internacional das doenças (CID 10). Porto Alegre: Sagra - DC - Luzatto, 1995.

17. Ornitz EM. The functional neuroanatomy of infantile autism. Inter J Neurosci. 1983,19:85-125.

18. Santana VS. Estudo epidemiológico das doenças mentais em um bairro de Salvador. Tese de Mestrado, Universidade Federal da Bahia. Salvador, 1978.

19. Schopler E, Reichler RJ, Devellis RF, Daly K. Toward objetive classification of childhood autism: childhood autism rating scale. J Autism Dev Disord 1980;10:91-103.

20. Schwartzman JS. Autismo infantil. Brasilia: Coordenadoria Nacional para Integração da Pessoa Portadora de Deficiência, 1994. 